\title{
Schätzung genetischer Parameter für Testtagsergebnisse der Milchleistung bei ostfriesischen Milchschafen mit Bayes-Methoden für longitudinale Daten
}

\author{
Herrn Prof. Drs. h. c. Franz Pirchner PhD, zum 75. Geburtstag gewidmet
}

\begin{abstract}
Summary
Title of the paper: Estimation of genetic parameters for test day results of milk performance in East Friesian milk sheep using Bayesian methods for longitudinal data

The objectives of this study were to estimate genetic parameters of milk performance traits in East Friesian milk sheep by using test day models with random regresssion. The analysis was based on 7545 test day records of 918 East Friesian milk sheep with 1380 lactation records. The data were provided by the sheep breeding organizations of Lower-Saxony, Westphalia, and Bavaria. The milk recordings were collected in the years 1992 to 2000. The average values of the heritability estimates by using random regression models were for the milk yield $h^{2}=0.25 \pm 0.03$, for the fat content $h^{2}=0.46 \pm 0.09$, and for the protein content $h^{2}=0.63 \pm 0.12$. The range of heritability estimates in dependence of the days in milk was for milk yield $h^{2}=0.03$ to 0.70 , for fat content $\mathrm{h}^{2}=0.30$ to 0.70 , and for protein content $\mathrm{h}^{2}=0.44$ to 0.92 .
\end{abstract}

Key Words: milk sheep, milk performance, random regression, heritability

\section{Zusammenfassung}

Ziel der vorliegenden Arbeit war es, die genetischen Parameter von Milchleistungsmerkmalen in Abhängigkeit von der Laktationsdauer mittels eines Random Regression Testtagstiermodells beim ostfriesischen Milchschaf zu analysieren. Für die Untersuchung standen 7545 Testtagsergebnisse von 918 milchleistungsgeprüften ostfriesischen Milchschafen mit 1380 Laktationsleistungen zur Verfügung. Die Milchleistungsdaten wurden von den Landesschafzuchtverbänden Niedersachsen, Weser-Ems, Westfalen-Lippe und der Bayerischen Herdbuchgesellschaft für Schafzucht bereitgestellt und stammten aus den Jahren 1992-2000. Die Mittelwerte der mittels des Testtagstiermodells mit zufälliger Regression geschätzten Heritabilitäten lagen für die Milchmenge, den Fettund Eiweißgehalt bei $h^{2}=0,25 \pm 0,03,0,46 \pm 0,09$ und 0,63 $\pm 0,12$. Im Verlauf der Laktation schwankten die Heritabilitäten bei der Milchmenge von $h^{2}=0,03$ bis 0,47 , beim Fettgehalt zwischen $h^{2}=0,30$ bis 0,70 und beim Eiweißgehalt zwischen $\mathrm{h}^{2}=0,44$ bis 0,92 .

Schlüsselwörter: Milchschafe, Milchleistung, Random Regression, Heritabilität

1. Einleitung

Die Zucht und Haltung von ostfriesischen Milchschafen hat in den letzten Jahren stark an Beliebtheit in Deutschland gewonnen. Die populationsgenetischen Analysen von Milchleistungsmerkmalen mit Tiermodellen und fixer Regression auf den Testtag zeigten, dass auch mit Daten aus der Milchleistungsprüfung auf Betrieben ein Zuchtfortschritt erreicht werden kann (HORSTICK et al., 2001). Die Weiterentwicklung des Testtagsmodells ermöglicht es, auch zufällige Effekte in die Schätzung der Regressionskurven aufzunehmen. Somit kann die möglicherweise unterschiedlich hohe Be- 
deutung der umweltdingten und additiv-genetischen Streuung im Verlauf der Laktation quantifiziert werden. Kovarianzfunktionen mit orthogonalen Polynomen eignen sich besonders gut dafür (BROTHERSTONE et al., 2000; KIRKPATRICK et al., 1994). Mit Hilfe dieser Kovarianzfunktionen können die Kovarianzen für alle zufälligen Effekte zwischen beliebigen Zeitpunkten der Laktation abgebildet werden. Das Ziel dieser Untersuchung ist es deshalb, die Heritabilitäten sowie die additiv-genetischen und residualen Varianz-Kovarianzmatrizen für die Milchleistungsmerkmale mittels Testtagstiermodellen mit zufälliger Regression (Random Regression) zu schätzen.

Das aus der Milchleistungsprüfung vorliegende Datenmaterial wurde von den Landesschafzuchtverbänden Niedersachsen, Weser-Ems, Westfalen-Lippe und der Bayerischen Herdbuchgesellschaft für Schafzucht zur Verfügung gestellt. Die Milchleistungsdaten von 918 Schafen umfassten 7545 Testtagsergebnisse aus 1380 Laktationen. Die Probemelkungen erfolgten fast ausschließlich nach der in den IKLT-Richtlinien beschriebenen Standardmethode (Methode A4), so dass bei jeder Probemelkung die Milchmenge pro Tag, der Fett- und Eiweißgehalt festgestellt wurden. Die Anzahl der geprüften Tiere und Testtagsergebnisse je Schafzuchtverband ist der Tabelle zu entnehmen. Die Probemelkergebnisse wurden in den Jahren 1992 bis einschließlich 2000 ermittelt. Die Testtagsergebnisse der Landesschafzuchtverbände Weser-Ems und Bayern lagen erst ab 1994 vor. Aus Westfalen-Lippe und Bayern gingen die Daten nur bis einschließlich dem Jahr 1999 ein. Die Tiere stammten aus insgesamt 90 Betrieben, in denen sie entweder von Hand oder maschinell gemolken wurden. 50,6 \% der Betriebe lagen im Kammergebiet Weser-Ems, 7,9 \% im Einzugsgebiet des Landesschafzuchtverbandes Niedersachsen, 13,5 \% im Bereich des Schafzuchtverbandes Westfalen-Lippe und 28,1 \% gehörten zur Bayerischen Herdbuchgesellschaft für Schafzucht. Zwischen den Betrieben gab es große Unterschiede in der Anzahl an Tieren, die an einer Milchleistungsprüfung teilnahmen.

Tabelle

Anzahl der geprüften Tiere, Laktationen und der Testtagsergebnisse (PM) nach Schafzuchtverbänden (Number of animals tested, lactations and test day results (PM) by sheep breeding organizations)

\begin{tabular}{lccc}
\hline Verband & Anzahl Tiere & Anzahl Laktationen & Anzahl PM \\
\hline Niedersachsen & 101 & 138 & 684 \\
Weser-Ems & 386 & 432 & 2396 \\
Westfalen-Lippe & 88 & 88 & 399 \\
Bayern & 34 & 722 & 4066 \\
\hline Gesamt & 918 & 1380 & 7545 \\
\hline
\end{tabular}

Die Anzahl der geborenen Lämmer variierte bei den geprüften Mutterschafen von 1 bis 5 Tieren, wobei am häufigsten Zwillinge (55,6 \% der Mutterschafe) und Drillinge (24,5 \% der Mutterschafe) auftraten. Die Milchleistungsergebnisse verteilten sich von der ersten bis zur elften Laktation. Im vorliegenden Datenmaterial erfolgte bei 37,7 \% der geprüften Schafe die Leistungsprüfung in der zweiten Laktation, bei 12,4 \% in der ersten und bei 49,9 \% in der dritten bis elften Laktation. Da Milchschafe eine saisonale Brunst zeigen, fand der Großteil der Ablammungen im Frühjahr in den Monaten Januar bis März (94,6 \%) statt. 
Von jedem geprüften Schaf wurde das Pedigree erhoben, so dass der Vater und die Mutter sowie das Geburtsdatum bekannt waren. Insgesamt stammten die Schafe von 261 Vätern und 630 Müttern ab, die sich wiederum auf 176 Großväter und 210 Großmütter väterlicherseits und 159 Großväter und 273 Großmütter mütterlicherseits zurückführen ließen. Die durchschnittliche Anzahl an Nachkommen eines Bockes, die in diese Untersuchung eingingen, lag bei 3,5. Im Datenmaterial waren 52 Großmütter und 203 Mütter enthalten, von denen sowohl das Ergebnis der eigenen Milchleistungsprüfung als auch Ergebnisse ihrer Nachkommen vorlagen.

Die Schätzung der additiv-genetischen Varianz-Kovarianzkomponenten der Milchleistungsparameter in Abhängigkeit von der Laktationsdauer erfolgte mittels eines Testtagstiermodells mit zufälliger Regression. Für die Schätzung der Kovarianzmatrix wurden Kovarianz-Funktionen herangezogen. Dafür wurden orthogonale Polynome vierter Ordnung verwandt. Da durch die orthogonalen Polynome vierter Ordnung nur noch 2,5 \% der geschätzten Varianz-Kovarianzstruktur erklärt wurde, war durch Polynome noch höherer Ordnung kein zusätzlicher Gewinn an Information zu erwarten. Der größte Anteil an den geschätzten Varianzen und Kovarianzen wurde über das Polynom erster (67,7 \%) und dritter (29,7 \%) Ordnung ermittelt. Die Auswertung erfolgte mit dem Programm DFREML, Version 3.0 $\beta$ (MEYER, 1998).

Modell 1 Testtagstiermodell mit zufälliger Regression für die Milchleistungsmerkmale der ostfriesischen Milchschafe

$$
\begin{aligned}
& \mathrm{y}_{\mathrm{ijklmnopqrs}}=\mu+\mathrm{R}_{\mathrm{i}}+\mathrm{S}_{\mathrm{j}}+\mathrm{J}_{\mathrm{k}}+\mathrm{Z}_{\mathrm{l}}+\mathrm{L}_{\mathrm{m}}+\mathrm{P}_{\mathrm{n}}+\mathrm{H}_{\mathrm{o}}\left[1 \mathrm{tt}^{2} \mathrm{t}^{3}\right] \\
& +\mathrm{b}_{1} \mathrm{TIM}_{\mathrm{p}}+\mathrm{b}_{2}\left(\mathrm{TIM}_{\mathrm{p}}\right)^{2}+\mathrm{b}_{3} \log \mathrm{TIM}_{\mathrm{p}}+\mathrm{b}_{4}\left(\log \mathrm{TIM}_{\mathrm{p}}\right)^{2} \\
& +\mathrm{p}_{\mathrm{q}}\left[1 \mathrm{t} \mathrm{t}^{2} \mathrm{t}^{3}\right] \\
& +\mathrm{a}_{\mathrm{r}}\left[1 \mathrm{t} \mathrm{t}^{2} \mathrm{t}^{3}\right] \\
& +\mathrm{e}_{\mathrm{ijklmnopqrs}} \\
& \mathrm{y}_{\mathrm{ijklmnopqrs}}=\text { beobachtetes Merkmal für die Milchmenge, den Fett- und } \\
& \text { Eiweißgehalt des ijklmnopqrs-tenTieres am Testtag } \\
& \mu \quad=\text { Modellkonstante } \\
& \mathrm{R}_{\mathrm{i}} \quad \text { = fixer Effekt der Region }(\mathrm{i}=1-4) \\
& \mathrm{S}_{\mathrm{j}} \quad=\text { fixer Effekt der Ablammsaison }(\mathrm{j}=1-3) \\
& \mathrm{J}_{\mathrm{k}} \quad \text { = fixer Effekt des Ablammjahres }(\mathrm{k}=1-8) \\
& \mathrm{Z}_{\mathrm{l}} \quad \text { = fixer Effekt der Anzahl geborener Lämmer }(\mathrm{l}=1-3) \\
& \mathrm{L}_{\mathrm{m}} \quad=\text { fixer Effekt der Laktationsnummer }(\mathrm{m}=1-5) \\
& \mathrm{P}_{\mathrm{n}} \quad=\text { fixer Effekt des Probemelk-Intervalls }(\cong \text { Testtag) }(\mathrm{n}=1-73) \\
& \mathrm{H}_{\mathrm{o}} \quad \text { = zufälliger Effekt der Herde }(\mathrm{o}=1-90) \\
& \mathrm{TIM}_{\mathrm{p}} \quad=\text { Tage in Milch als Kovariable } \\
& \mathrm{b}_{1}-\mathrm{b}_{4} \quad \text { = fixe Regressionskoeffizienten } \\
& \mathrm{p}_{\mathrm{q}} \quad=\text { zufälliger permanenter Umwelteffekt des Tieres } \\
& \mathrm{a}_{\mathrm{r}} \quad=\text { zufälliger additiv-genetischer Effekt des Tieres } \\
& \mathrm{t} \quad=\text { Tage in Milch } \\
& {\left[1 \mathrm{t} \mathrm{t}^{2} \mathrm{t}^{3}\right] \quad=\text { Vektoren der zufälligen Regressionskoeffizienten }} \\
& \mathrm{e}_{\mathrm{ijklmnopqrs}} \quad=\text { zufälliger Restfehler }
\end{aligned}
$$


3. Ergebnisse

Die Abbildungen 1 bis 3 zeigen die mittels des Testtagstiermodells mit zufälliger Regression für jeden einzelnen Laktationstag geschätzten additiv-genetischen Varianzen und Kovarianzen. Dabei sind die Änderungen der Varianz und Kovarianz als Funktion der Zeit dargestellt. Die Diagonale der Grafik repräsentiert die additiv-genetische Varianz des Merkmals an jedem einzelnen Laktationstag, wogegen jeweils beiderseits der Diagonalen die additiv-genetische Kovarianz zwischen den einzelnen Laktationstagen aufgetragen ist. Die mittels Random Regression geschätzte additiv-genetische Varianz der Milchmenge lag zwischen $\mathrm{s}_{\mathrm{a}}{ }^{2}=0,023$ und 0,392. Zunächst fiel die additiv-genetische Varianz bis zum 20. Laktationstag auf das Minimum von $\mathrm{s}_{\mathrm{a}}^{2}=$ 0,023 ab. Ab etwa dem 30. Tag in Milch stieg die Varianz bis zum etwa 150. Laktationstag stetig an und erreichte dabei einen Wert von $\mathrm{s}_{\mathrm{a}}^{2}=0,189$. In dieser Höhe blieb der Schätzwert bis zum 170. Laktationstag. Danach kam es zu einem Abfall der Varianz bis auf $\mathrm{s}_{\mathrm{a}}^{2}=0,121 \mathrm{um}$ den 260. Laktationstag. In den letzten Laktationstagen stieg die additiv-genetische Varianz der Milchmenge abermals an und erreichte beim letzten bei der Auswertung berücksichtigten Melktag die maximale Varianz von s ${ }_{a}^{2}=0,392$. Die additiv-genetischen Kovarianzen zwischen den einzelnen Laktationstagen für die Milchmenge lagen in einem Bereich von $-0,2$ bis 0,5, woraus ersichtlich wurde, dass die Kovarianzen zwischen den verschiedenen Laktationstagen deutlichen Schwankungen unterlagen (Abb. 1).

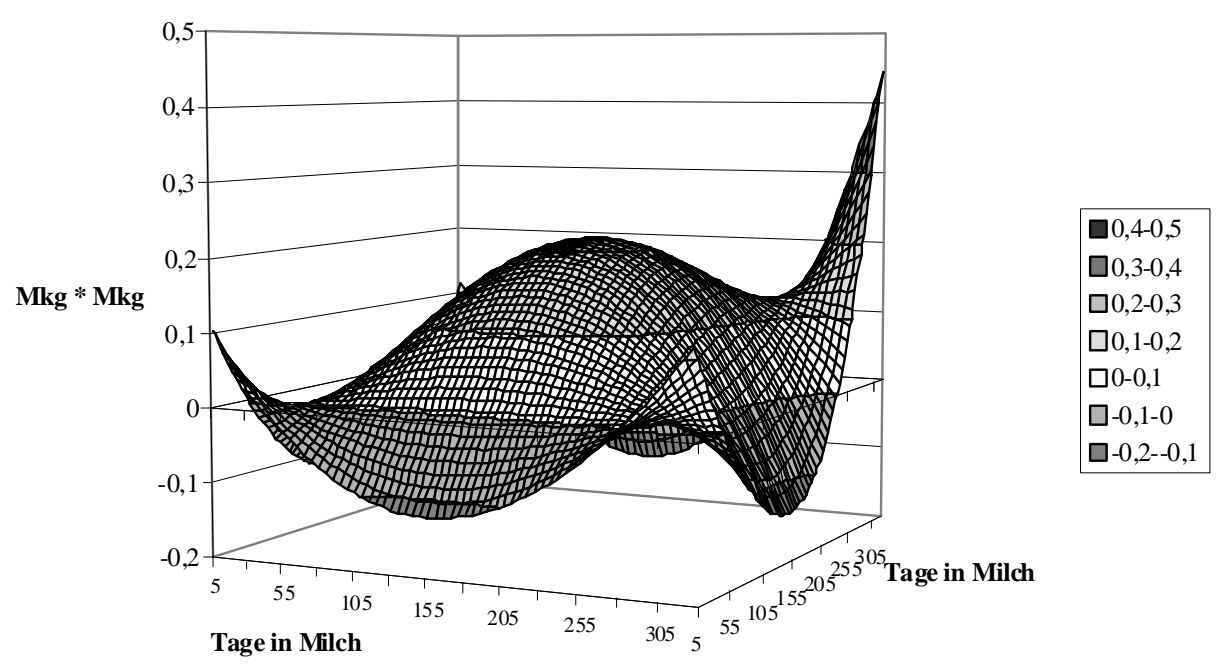

Abb. 1: Additiv-genetische Kovarianz-Funktionen der Milchmenge in Abhängigkeit von dem Laktationstag (Additive genetic covariance functions of milk yield in dependence of the day of lactation)

Die additiv-genetische Varianz des Fettgehaltes der Milch der ostfriesischen Milchschafe lag zu Beginn der Laktation in einem sehr hohen Bereich von über $\mathrm{s}_{\mathrm{a}}{ }_{\mathrm{a}}=1,0$. Bis zum 60. Laktationstag sank die Varianz stetig ab und pendelte sich von diesem Zeitpunkt an auf einen Schätzwert von annähernd $\mathrm{s}^{2}{ }_{\mathrm{a}}=0,5$ ein. Etwa ab dem 190. Tag der Laktation stieg die Varianz bis zum Laktationsende wieder an und erreichte dabei die maximale additiv-genetische Varianz des Fettgehaltes von $\mathrm{s}_{\mathrm{a}}{ }_{\mathrm{a}}=2,98$. Die Kovarianzen zwischen den einzelnen Laktationstagen für den Fettgehalt lagen zwischen -1,49 und 2,98. Zeitlich nah zusammenliegende Laktationstage wiesen dabei die höchsten Kovarianzen auf, im Gegensatz zu Tagen, die weit voneinander entfernt lagen. Für diese wurden die niedrigsten additiv-genetischen Kovarianzen geschätzt (Abb. 2). 


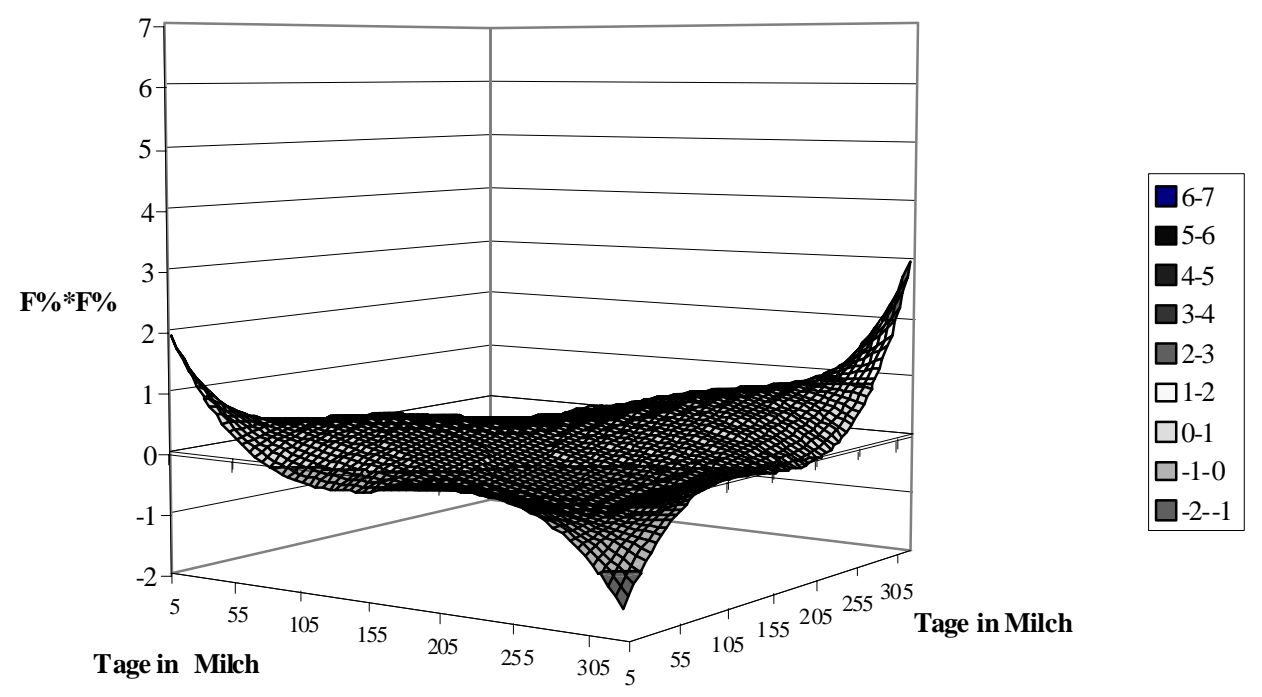

Abb. 2: Additiv-genetische Kovarianz-Funktionen für den Fettgehalt in Abhängigkeit von dem Laktationstag (Additive genetic covariance functions of the fat content in dependence of the day of lactation)

Auch die additiv-genetischen Varianzen des Eiweißgehaltes der ostfriesischen Milchschafe fielen zunächst nach einem hohen Ausgangswert auf ein Minimum von $\mathrm{s}_{\mathrm{a}}=$ 0,567 um den 40. Laktationstag ab. Danach stieg die additiv-genetische Varianz kontinuierlich bis zum Laktationsende sehr langsam an, bis dann eine maximale Varianz von $\mathrm{s}_{\mathrm{a}}{ }_{\mathrm{a}}=6,92$ erreicht wurde. Die geschätzten Kovarianzen variierten zwischen -1,2 und 6,92, wobei auch hier, ebenso wie beim Fettgehalt, für dicht beieinander liegende Laktationstage die höchsten Kovarianzen und für weit von einander entfernt liegende Laktationstage die niedrigsten Kovarianzen geschätzt wurden (Abb. 3).

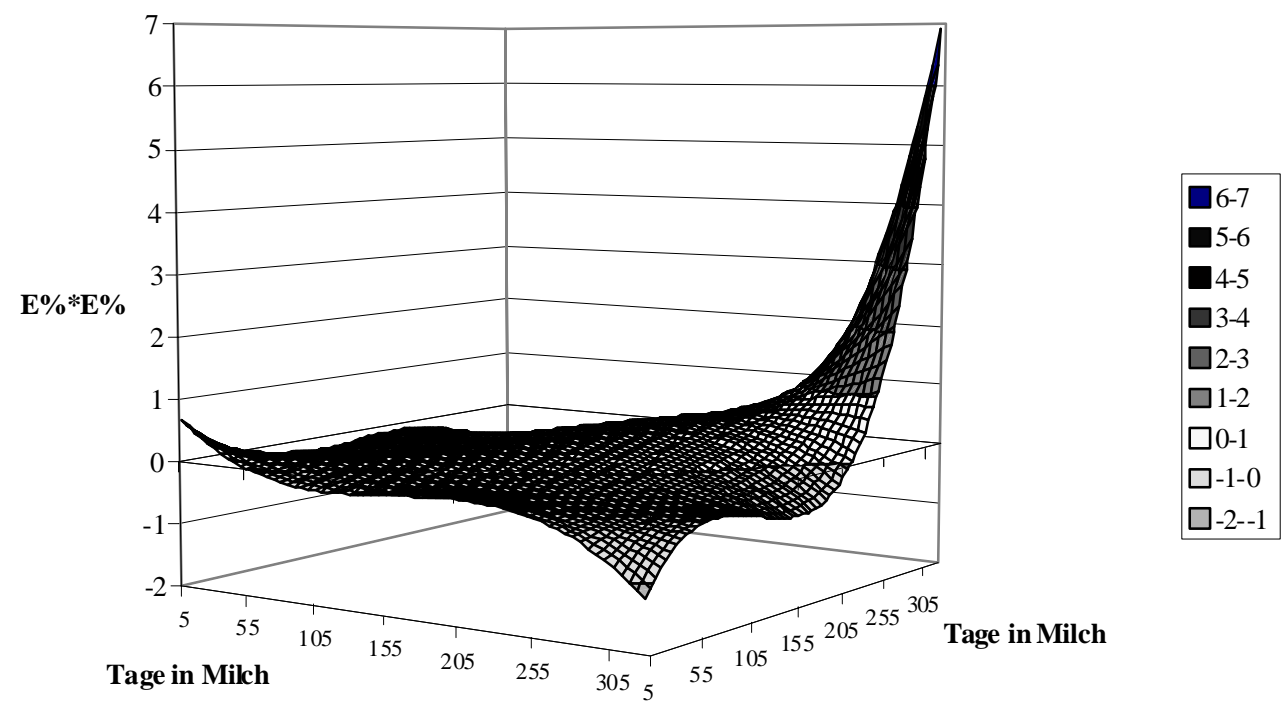

Abb. 3: Additiv-genetische Kovarianz-Funktionen für den Eiweißgehalt in Abhängigkeit von dem Laktationstag (Additive genetic covariance functions of the protein content in dependence of the day of lactation)

Die mittels Random Regression geschätzten mittleren Heritabilitäten der Milchmenge für jeden einzelnen Laktationstag betrugen $h^{2}=0,25 \pm 0,03$. Die Schwankungsbreite lag zwischen $\mathrm{h}^{2}=0,03$ bis 0,47 . In den ersten Laktationstagen erreichte die Heritabilität einen Wert von $\mathrm{h}^{2}=0,03$, der jedoch bis zum 25. Tag auf die Hälfte des Ausgangs- 
wertes absank. Danach stieg die Heritabilität auf einen maximalen Wert von $\mathrm{h}^{2}=0,47$ am 183. Laktationstag an. Anschließend fiel sie bis zum 270. Tag auf einen Wert von $h^{2}=0,25$ ab. Nach dem 270. Tag kam es nochmals zu einem deutlichen Anstieg der Heritabilität, wobei diese Schätzwerte nur noch auf einem geringen Datenmaterial beruhten. Die mittels eines Testtagstiermodells mit fixer Regression geschätzten Heritabilitäten von $\mathrm{h}^{2}=0,18$ lagen hier nur annähernd zwischen dem 77 . bis 82. Laktationstag vor.

Die Heritabilitäten des Fettgehaltes der ostfriesischen Milchschafe schwankten während einer Laktation zwischen $\mathrm{h}^{2}=0,30$ und $\mathrm{h}^{2}=0,70$ mit einem Mittelwert von $\mathrm{h}^{2}=$ $0,46 \pm 0,09$. Zu Beginn der Laktation erreichte die Heritabilität einen Schätzwert von $\mathrm{h}^{2}=0,50$, der jedoch innerhalb des ersten Laktationsmonats auf einen Wert von $\mathrm{h}^{2}=$ 0,30 absank. Danach stieg die Heritabilität im Gegensatz zur Milchmenge bis zum Laktationsende an. Dabei bewegten sich die Schätzwerte zwischen dem 63. und 182. Laktationstag bei $\mathrm{h}^{2}=0,40$. Danach stieg die Heritabilität bis auf Werte von $\mathrm{h}^{2}=0,56$ an. Ab dem 290. Tag kam es nochmals zu einem Anstieg der Heritabilität bis auf $\mathrm{h}^{2}=$ 0,70 , wobei auch hier nur noch eine geringe Datenmenge zur Verfügung stand.

Der Verlauf der Heritabilitäten des Eiweißgehalts ist mit der Kurve des Fettgehalts vergleichbar. Insgesamt waren die geschätzten Heritabilitäten etwas höher, sie schwankten zwischen $\mathrm{h}^{2}=0,44$ und $\mathrm{h}^{2}=0,92$ bei einem Mittelwert von $\mathrm{h}^{2}=0,63 \pm$ 0,12. Auch beim Eiweißgehalt zeigte sich zunächst ein Absinken der Heritabilität innerhalb der ersten 30 Laktationstage auf den niedrigsten Schätzwert der gesamten Laktation. Etwa ab dem 50. Tag stieg die Heritabilität stetig bis zum Laktationsende an. Am Laktationsende waren auch beim Eiweißgehalt die Heritabilitätsschätzwerte am höchsten.

4. Diskussion

Die Schätzung der Heritabilitäten und additiv-genetischen Korrelationen kann durch unterschiedliche Faktoren beeinflusst werden. Zunächst können dies die Methode der Schätzung und die dabei berücksichtigten genetischen Effekte sein. Weiterhin übt die zugrundeliegende Datenmenge und Datenstruktur einen erheblichen Einfluss auf das Schätzergebnis aus. Bei der Analyse von Milchleistungsmerkmalen muss insbesondere zwischen der Auswertung von Testtagsergebnissen und der Gesamtlaktationsleistung unterschieden werden. Bei Testtagsergebnissen ist der Messfehler pro Beobachtung größer, so dass deshalb eine niedrigere Heritabilität zu erwarten ist. Bei den Testtagsmodellen ist zu unterscheiden, ob nur fixe oder auch zufällige Regressionsterme verwendet werden. Random Regression Modelle sollten für die Analyse von longitudinalen Daten, wie z.B. für Probemelkergebnisse, besser angepasst als fixe Regressionsmodelle sein, da sie auch die zufälligen Modellkomponenten für jeden Testtag berücksichtigen können. Jedoch können bei Random Regression Modellen zu Beginn und am Ende der Laktation insbesondere dann Überschätzungen der Varianzkomponenten auftreten, wenn wesentliche zufällige Effkte in der Schätzfunktion für den Testtag vernachlässigt werden (KETTUNEN et al., 2000). Deshalb wurden in der vorliegenden Untersuchung alle zufälligen Effekte als zufällige Regressionskoeffizienten im Random Regression Modell berücksichtigt.

Die Schätzungen der Heritabilität mittels des Testtagstiermodells mit zufälliger Regression ergaben für die ostfriesischen Milchschafe deutlich höhere Heritabilitäten als mit uni- oder multivariaten Modellen mit fixer Regression (HORSTICK et al., 2001). 
In den letztgenannten Analysen bewegten sich die Heritabilitäten für die Milchleistungsmerkmale mit Ausnahme des Eiweißgehalts zwischen $h^{2}=0,1$ und 0,2. Für den Eiweißgehalt ergaben sich Heritabilitätsschätzwerte von $\mathrm{h}^{2}=0,24$. Als Grund für die höheren Heritabilitätsschätzwerte im Random Regression Modell ist anzuführen, dass die Berücksichtigung der zufälligen Regressionskomponenten im Testtagstiermodell eine bessere Trennung des additiv-genetischen Effektes des Tieres von den Effekten der Herde und der permanenten Umwelt ermöglichte. Das Testtagstiermodell mit zufälliger Regression neigte auch in diesem Modellansatz dazu, am Ende der Laktation den zufälligen additiv-genetischen Effekt des Tieres höher zu schätzen als die durch die Herde verursachte Varianz. Der bei allen mittels des Testtagstiermodells mit zufälliger Regression untersuchten Merkmalen am Ende der Laktation festzustellende Anstieg der additiv-genetischen Varianz und der Heritabilitäten ist auf die Kovarianzfunktion zurückzuführen, und dieses könnte wiederum mit der geringeren Anzahl von Probemelkergebnissen von Milchschafen am Ende der Laktation zusammenhängen. Der am Ende der Laktation bei allen analysierten Merkmalen vorhandene Anstieg der Heritabilitäten spricht, wie bei den Analysen bei den Ayrshire Rindern in Finnland (KETTUNEN et al., 2000), für eine gewisse Überschätzung. Allerdings war die mögliche Überschätzung der Heritabilität am Ende der Laktation bei den Milchschafen nicht so drastisch wie in den Analysen bei den Ayrshire Kühen. Die durchschnittlichen Heritabilitätsschätzwerte aus den Random Regression Modellen stimmten annähernd mit den Werten von BARILLET und BOICHARD (1987) für die Milchleistungsmerkmale, berechnet als standardisierte Laktationsleistung, bei Lacaune Schafen überein. Dagegen schätzten BARO et al. (1994) mit einem Testtagstiermodell und fixer Regression bei spanischen Churra-Schafen sowie MAVROGENIS und PAPACHRISTOFOROU (2000) bei zypriotischen Chios Schafen höhere Heritabilitäten für die Milchmenge, während in den Untersuchungen von CARRIEDO et al. (1995) und EL-SAIED et al. (1998) deutlich niedrigere Heritabilitätsschätzwerte für Laktationsleistungen der Milchmenge gefunden wurden.

Mittels der Random-Regression-Testtagstiermodelle ist eine Trennung der additiv-genetischen Varianz von der Varianz der permanenten Umwelt und des Besitzers im Verlauf der Laktation möglich, so dass die genetisch bedingte Reaktion des Tieres auf die Umwelt bei der Schätzung genetischer Parameter besser berücksichtigt werden kann. Jedoch sollten die Ergebnisse sorgfältig daraufhin geprüft werden, ob möglicherweise zu Beginn und/oder zu Ende der Laktation eine Überschätzung der additivgenetischen Varianzen vorliegt.

\section{Danksagung}

Der H. Wilhelm Schaumann Stiftung, Hamburg, sei für die Unterstützung dieser Arbeit sehr herzlich gedankt.

\section{Literatur}

BARILLET, F.; BOICHARD, D.:

Studies on dairy production of milking ewes. 1. Estimates of genetic parameters for total milk composition and yield. Génét. Sél. Evol. 19 (1987), 459-474

BARO, J.A.; CARRIEDO, J.A.; SAN PRIMITIVO, F.:

Genetic parameters of test day measures for somatic cell count, milk yield and protein percentage of milking ewes. J. Dairy Sci. 77 (1994), 1658-2662 
BROTHERSTONE, S.; WHITE, I.M.S.; MEYER, K.:

Genetic modelling of daily milk using orthogonal polynomials and parametric curves. Anim. Sci. 70 (2000), 407-415

CARRIEDO, J.A., BARO, J.A., DE LA FUENTE, L.F., SAN PRIMITIVO, F.:

Genetic parameters for milk yield in dairy sheep. J. Anim. Breed. Genet. 112 (1995), 59-63

EL-SAIED, M.U.; CARRIEDO, J.A.; SAN PRIMITIVO, F.:

Heritability of test day somatic cell counts and its relationship with milk yield and protein percentage in dairy ewes. J. Dairy Sci. 81 (1998), 2956-2961

HORSTICK, A.; HAMANN, H.; DISTL, O.:

Analyse von Milchleistungsmerkmalen bei ostfriesischen und schwarzbraunen Milchschafen. Züchtungskunde, Stuttgart 73 (2001), 343-352

KETTUNEN, A.; MÄNTYSAARI, E.A.; PÖSÖ, J.:

Estimation of genetic parameters for daily milk yield of primiparous Ayrshire cows by random regression test-day models. Livest. Prod. Sci. 66 (2000), 251-261

KIRKPATRICK, M.; HILL, W.G.; THOMPSON, R.:

Estimating covariance structure of traits during growth and ageing, illustrated with lactation in dairy cattle. Genet. Res. 64 (1994), 57-69

MAVROGENIS, A.P.; PAPACHRISTOFOROU, C.:

Genetic and phenotypic relationships between milk production and body weight in Chios sheep and MEYER, K.: Damascus goats. Livest. Prod. Sci. 67 (2000), 81-87

Modelling ,repeated' records: Covariance functions and random regression models to analyse animal breeding data. Proceedings of the $6^{\text {th }}$ World Congress on Genetics Applied to Livestock Production Vol. 25 (1998), 517-520

Eingegangen: 05.07.2001

Akzeptiert: 20.12.2001

Anschrift der Verfasser

DR. ANNETTE HORSTICK, Prof. Dr. OTTMAR DISTL

Institut für Tierzucht und Vererbungsforschung

Bünteweg 17p

D-30559 Hannover

E-Mail: ottmar.distl@tiho-hannover.de 